\title{
Energy-Efficient Resource Allocation for NOMA-based Backscatter Communications
}

\author{
Yongjun Xu, Member, IEEE, Zhijin Qin, Member, IEEE, and Guan Gui, Senior Member, IEEE
}

\begin{abstract}
Backscatter communication (BackCom) is a promising technique for achieving high spectrum efficiency and power efficiency in the future Internet of Things systems. The capacity of BackCom networks can be maximized by optimizing the backscatter time and the reflection coefficient (RC). However, system energy efficiency (EE) cannot be guaranteed usually. In this paper, we investigate the energy-efficient resource allocation problem of a non-orthogonal multiple access (NOMA)-based BackCom. Particularly, the base station (BS) transmits signals to two cellular users based on the NOMA protocol, meanwhile, a backscatter device backscatters the signals to users using the passive radio technology. The total EE of the considered system is maximized by jointly optimizing power allocation for each NOMA user and the RC of backscatter device where the decoding order and the quality of service $(\mathrm{QoS})$ of each user are guaranteed. To solve such a non-convex problem, we develop an efficient iterative algorithm to obtain the optimal solutions by using Dinkelbach's method and the quadratic transformation approach. Numerical results show that the proposed algorithm can significantly improve the system EE compared with the orthogonal multiple access (OMA) scheme and the NOMA system without backscatter devices.
\end{abstract}

Index Terms-Backscatter communications, NOMA, resource allocation, energy efficiency.

\section{INTRODUCTION}

$\mathbf{E}$ NERGY consumption is a critical issue for the fifth generation $(5 \mathrm{G})$ wireless communication systems [1], which should be addressed in the future systems. Backscatter communications (BackComs) [2], [3] have been considered as a candidate to extend the lifetime of IoT sytems by directly reflecting the incident radio frequency (RF) signals to the indicated receivers without modulating or generating RF signals by itself. Therefore, BackCom could enhance the signal quality received at the users and reduce the energy consumption of system. Moreover, to provide more access opportunities for massive number of IoT users, non-orthogonal multiple access (NOMA) [4], [5] can be adopted by allowing multiple users to share the same resource block.

To overcome the near-far problem in communication systems, NOMA-based BackCom systems have been investigated to improve system capacity and spectrum utilization [6]. In [7], the closed-form expression of the outage probability and the ergodic rate of NOMA users have been provided for a downlink NOMA-based BackCom system. In [8],

Y. Xu is with School of Communication and Information Engineering, Chongqing University of Posts and Telecommunication, Chongqing, China. Z. Qin is with School of Electronic Engineering and Computer Science, Queen Mary University of London, London, UK. G. Gui is with College of Telecommunications and Information Engineering, Nanjing University of Posts and Telecommunications, Nanjing 210003, China. the outage probability has been derived for a NOMA-based BackCom system where the base station (BS) is equipped with multiple antennas. In [9], the exact analytical closedform expression for the fading-free scenario and semi closedform expression for the fading scenario have been derived for an uplink NOMA-based BackCom system. However, the above works focus on the performance analysis of the system (e.g., outage probability expression). In [10], the resource allocation (RA) problem for a NOMA-based BackCom system has been formulated as a max-min throughput problem and solved by jointly optimizing the backscatter time and reflection coefficients (RCs). The suboptimal solution has been obtained by using the block coordinated decent and successive convex optimization approaches.

Although the aforementioned works [6]-[10] have laid solid contributions for understanding BackCom systems from various perspectives, e.g., performance analysis and throughput maximization, the optimal solutions of the RA problems have not been obtained yet. Moreover, the decoding order of NOMA user is only considered under the quasi-static channel relationship. Due to the coupled relationship of RC and channel coefficients, however, the effect of dynamic RC is not reflected in the decoding order of NOMA. To the best of our knowledge, this is the first paper to consider the EEbased RA problem for a downlink NOMA-based BackCom system. The main contribution of this paper is summarized as follows: (i) We formulated the RA in NOMA-based BackCom systems as an EE maximization problem by jointly optimizing the transmit power of BS and the RC of the backscatter device (BD), which considers the the minimum signal-to-interference plus-noise ratio (SINR) required by each NOMA user and the decoding order constraint. (ii) The non-convex and fractional optimization problem is converted into an equivalent problem based on the Dinklbach's method. The difference of concave function (DC) problem is transformed into the convex format by using a quadratic transformation approach. The closed-form solutions are obtained by using the Lagrange dual method and the subgradient approach.

\section{System Model and Problem Formulation}

As illustrated in Fig. 1, we consider a downlink NOMAbased BackCom system. By considering the hardware complexity of successful interference cancellation (SIC), we take two-user case as an example [11], [12]. The BS with the single antenna transmits the superposition signal to user 1 (i.e., the nearby user with good channel condition) and user 2 (i.e., the far-away user with poor channel condition) in the 


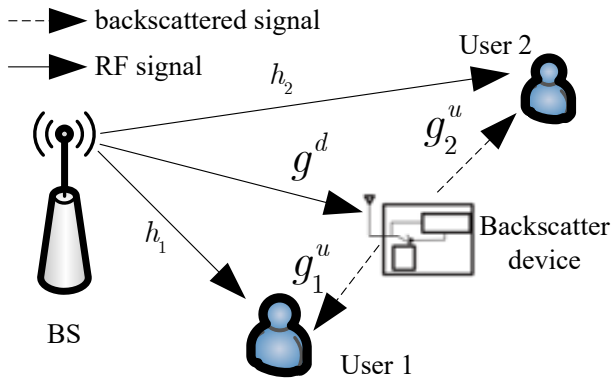

Fig. 1. System model of downlink BackCom with two NOMA users.

same resource block by assigning them with different transmit power. The BD reflects the signal received from the BS to user 1 and user 2. At user 1, it decodes the user 2's signal first, then decodes its own signal, which is achieved by SIC. Here, we assume that the BS can obtain perfect channel state information (CSI) by channel training [6]-[10].

\section{A. Received Signals of Users}

The transmitted complex signal from the BS can be written as

$$
x(t)=\sqrt{p_{1}} x_{1}(t)+\sqrt{p_{2}} x_{2}(t),
$$

where $x_{1}(t)$ and $x_{2}(t)$ are the transmitted signal from the BS to user 1 and user 2 , respectively, and satisfying $\mathbb{E}\left[\left|x_{1}(t)\right|^{2}\right]=$ 1 and $\mathbb{E}\left[\left|x_{2}(t)\right|^{2}\right]=1$. We denote $p_{1}$ and $p_{2}$ as the transmit power allocated to user 1 and user 2 , respectively.

User 1 receives two types of signals including the direct link signal from the BS and the backscatter signal from the BD. Therefore, the signals received at user 1 can be expressed as

$$
y_{1}(t)=\sqrt{h_{1}} x(t)+\sqrt{\rho g^{d} g_{1}^{u}} x(t) c(t)+n_{1}(t),
$$

where $h_{1}$ and $g^{d}$ denote the channel gain from the BS to user 1 and $\mathrm{BD}$, respectively, $g_{1}^{u}$ denotes the channel gain from the BD to user $1, \rho$ denotes the RC of the BD, which can be dynamically adjusted by the capacitor circuit [2]. $c(t)$ denotes the own message of the $\mathrm{BD}$ and satisfies $\mathbb{E}\left[|c(t)|^{2}\right]=1$, and $n_{1}(t)$ denotes the complex Gaussian noise at user 1 and satisfies $n_{1}(t) \sim \mathcal{C N}\left(0, \sigma^{2}\right)$.

According to SIC, user 1 can decode the signal of user 2, i.e., $x_{2}(t)$. The SINR at which user 1 decodes the signal of user 2 can be given by

$$
\gamma_{1 \rightarrow 2}=\frac{p_{2}\left(h_{1}+\rho g^{d} g_{1}^{u}\right)}{p_{1}\left(h_{1}+\rho g^{d} g_{1}^{u}\right)+\sigma^{2}},
$$

If the decoding succeeds, user 1 invokes the classic SIC and decodes its own message. The received SINR at user 1 can be expressed as

$$
\gamma_{1 \rightarrow 1}=\frac{p_{1}\left(h_{1}+\rho g^{d} g_{1}^{u}\right)}{\sigma^{2}} .
$$

Similarly, the received signals at user 2 is

$$
y_{2}(t)=\sqrt{h_{2}} x(t)+\sqrt{\rho g^{d} g_{2}^{u}} x(t) c(t)+n_{2}(t),
$$

where $h_{2}$ and $g_{2}^{u}$ denote the channel gain from the BS to user 2 and from the $\mathrm{BD}$ to user 2 , respectively, and $n_{2}(t)$ denotes the complex Gaussian noise at the user 1 and satisfies $n_{2}(t) \sim \mathcal{C N}\left(0, \sigma^{2}\right)$. Moreover, channel condition is assumed to satisfy $h_{1}>h_{2}$ [13]. As for user 2 , it will directly decode its own message by treating the message of user 1 as the interference. As a result, the received SINR at user 2 can be expressed as

$$
\gamma_{2 \rightarrow 2}=\frac{p_{2}\left(h_{2}+\rho g^{d} g_{2}^{u}\right)}{p_{1}\left(h_{2}+\rho g^{d} g_{2}^{u}\right)+\sigma^{2}} .
$$

\section{B. Problem Formulation}

When user 1 desires to decode the signal of user 2 , the interference cancellation is successful if user 1 's received SINR is no less than user 2's own received SINR [14]. Thus, to protect the given SIC decoding order, there must be a decoding SINR constraint

$$
\gamma_{1 \rightarrow 2} \geq \gamma_{2 \rightarrow 2}
$$

Thus, the total EE-based maximization RA can be formulated as

$$
\begin{array}{ll}
\text { P1 } & \max _{p_{i}, \rho} \frac{\log _{2}\left(1+\gamma_{1 \rightarrow 1}\right)+\log _{2}\left(1+\gamma_{2 \rightarrow 2}\right)}{\sum_{i=1}^{2} p_{i}+P_{c}} \\
\text { s.t. } & C_{1}: 0 \leq \rho \leq 1, C_{2}: \sum_{i=1}^{2} p_{i} \leq P, \\
& C_{3}: 0 \leq p_{i}, i=1,2, C_{4}: \gamma_{i} \geq \gamma_{i}^{\min }, i=1,2, \\
& C_{5}: \gamma_{1 \rightarrow 2} \geq \gamma_{2 \rightarrow 2},
\end{array}
$$

where $P_{c}$ denotes the total circuit power consumption, $P$ denotes the maximum transmit power at the BS, and $\gamma_{i}^{\min }$ is the minimum SINR requirement of each NOMA user. $C_{1}$ denotes the RC constraint. $C_{2}$ and $C_{3}$ are the constraints which determine the upper bound of transmit power. $C_{4}$ denotes the minimum SINR requirement for each NOMA user. $C_{5}$ denotes the decoding order constraint. P1 is a non-convex optimization problem due to the coupled variables $p_{i}$ and $\rho$. It is noted that $C_{1}-C_{3}$ are convex. We only need to focus on the transformation of $C_{4}, C_{5}$ and the objective function due to the coupled variables.

\section{Resource Allocation Algorithm Design}

In this section, we first derive the optimal RC according to the monotonicity of the objective function. Then an iterative algorithm is proposed to obtain the optimal transmit power.

\section{A. Optimal Reflection Coefficient}

We introduce $\bar{g}_{1}=g^{d} g_{1}^{u}$ and $\bar{g}_{2}=g^{d} g_{2}^{u}$ for symbol simplification. By using Dinkelbach's method, the objective function in $\mathbf{P 1}$ can be converted into a subtractive form. Thus P1 can be transformed into

$$
\begin{aligned}
& \text { P2 } \max _{p_{1}, p_{2}, \rho} \log _{2}\left(1+\frac{p_{1}\left(h_{1}+p_{1} \rho \bar{g}_{1}\right)}{\sigma^{2}}\right)-\eta\left(\sum_{i=1}^{2} p_{i}+P_{c}\right) \\
& +\log _{2}\left(1+\frac{p_{2}\left(h_{2}+\rho \bar{g}_{2}\right)}{p_{1}\left(h_{2}+\rho \bar{g}_{2}\right)+\sigma^{2}}\right) \\
& \text { s.t. } C_{1}-C_{3}, C_{5}: h_{1}-h_{2} \geq \rho g^{d}\left(g_{2}^{u}-g_{1}^{u}\right) \text {, } \\
& C_{6}: \frac{p_{1}\left(h_{1}+\rho \bar{g}_{1}\right)}{\sigma^{2}} \geq \gamma_{1}^{\min },
\end{aligned}
$$




$$
C_{7}: \frac{p_{2}\left(h_{2}+\rho \bar{g}_{2}\right)}{p_{1}\left(h_{2}+\rho \bar{g}_{2}\right)+\sigma^{2}} \geq \gamma_{2}^{\min },
$$

where $\bar{C}_{5}$ can be obtained by substituting (3), (6) into $C_{5}$. $C_{6}$ and $C_{7}$ can be obtained by substituting (4), (6) into $C_{4}$. Even though the problem is more tractable, there are coupling relationships among different optimization variables. In order to solve it, we first present the following Proposition.

Proposition 1: For any given system setting and optimization variable, the optimal $R C, \rho^{*}$, of $\mathbf{P 2}$ is calculated as

$$
\rho^{*}=\min \left(1, \max \left(0, \frac{h_{1}-h_{2}}{g^{d}\left(g_{2}^{u}-g_{1}^{u}\right)}\right)\right) .
$$

Proof: Please see Appendix A.

Remark 1: The proposed Proposition 1 serves two purposes. Firstly, we provide a closed-form expression for the optimal $\mathrm{RC}$ and hence get the optimal RC using the above closed-form expresssion without requiring any iterative algorithms, which reduce the computational complexity significantly. Secondly, we can get more insights about on the optimal RC. For example, when $\rho^{*}=1$, the optimal RC can be obtained when the BD is close to user 1 , namely, $h_{1}+g^{d} g_{1}^{u}>h_{2}+g^{d} g_{2}^{u}$ from $\bar{C}_{5}$. Since the data rate of user 1 has no co-channel interference in (4), it can further increase the sum rate of users. When $\rho^{*}=\left(h_{1}-h_{2}\right) /\left(g^{d}\left(g_{2}^{u}-g_{1}^{u}\right)\right), g_{2}^{u}>g_{1}^{u}$ means that the BD is closer to user 2 .

\section{B. Optimal Power Allocation}

When $\rho=1$, we obtain P3. When $\rho=$ $\left(h_{1}-h_{2}\right) /\left(g^{d}\left(g_{2}^{u}-g_{1}^{u}\right)\right)$, we obtain P4. According to the proposed Proposition 1, we denote $h=\rho=$ $h_{1}-h_{2} /\left(g\left(g_{2}-g_{1}\right)\right)$. Then $\mathbf{P 2}$ can be equivalent to the following two power allocation optimization problems, i.e.,

$$
\begin{aligned}
& \mathbf{P 3} \max _{p_{1}, p_{2}} \log _{2}\left(1+\frac{p_{1}\left(h_{1}+\bar{g}_{1}\right)}{\sigma^{2}}\right)-\eta\left(\sum_{i=1}^{2} p_{i}+P_{c}\right) \\
& +\log _{2}\left(1+\frac{p_{2}\left(h_{2}+\bar{g}_{2}\right)}{p_{1}\left(h_{2}+\bar{g}_{2}\right)+\sigma^{2}}\right) \\
& \text { s.t. } C_{2}-C_{3}, C_{6}: \frac{p_{1}\left(h_{1}+\bar{g}_{1}\right)}{\sigma^{2}} \geq \gamma_{1}^{\min }, \\
& \quad C_{7}: \frac{p_{2}\left(h_{2}+\bar{g}_{2}\right)}{p_{1}\left(h_{2}+\bar{g}_{2}\right)+\sigma^{2} \geq \gamma_{2}^{\min },} \\
& \text { P4 } \max _{p_{1}, p_{2}} \log _{2}\left(1+\frac{p_{1}\left(h_{1}+h \bar{g}_{1}\right)}{\sigma^{2}}\right)-\eta\left(\sum_{i=1}^{2} p_{i}+P_{c}\right) \\
& +\log _{2}\left(1+\frac{p_{2}\left(h_{2}+h \bar{g}_{2}\right)}{p_{1}\left(h_{2}+h \bar{g}_{2}\right)+\sigma^{2}}\right) \\
& \text { s.t. } C_{2}-C_{3}, \tilde{C}_{6}: \frac{p_{1}\left(h_{1}+h \bar{g}_{1}\right)}{\sigma^{2}} \geq \gamma_{1}^{\min }, \\
& \tilde{C}_{7}: \frac{p_{2}\left(h_{2}+h \bar{g}_{2}\right)}{p_{1}\left(h_{2}+h \bar{g}_{2}\right)+\sigma^{2} \geq \gamma_{2}^{\min },}
\end{aligned}
$$

It is noted that $\mathbf{P 3}$ and $\mathbf{P 4}$ have the same structure in which there are the concave objective function and linear constraints except for the second item of the objective function.

Proposition 2: For any given system settings, define $\bar{h}_{2}=h_{2}+h \bar{g}_{2}$, the function $G\left(p_{1}, p_{2}\right)=$ $\log _{2}\left(1+p_{2} \bar{h}_{2} /\left(p_{1} \bar{h}_{2}+\sigma^{2}\right)\right)$ is a non-convex or non-concave function.

Proof: Please see Appendix B.

In order to make the problem traceable, by applying the quadratic transformation [15], P3 and P4 can be converted into the convex form as

$$
\begin{gathered}
\text { P5 } \max _{p_{1}, p_{2}, y} \log _{2}\left(1+\frac{p_{1} \bar{h}_{1}}{\sigma^{2}}\right)-\eta\left(\sum_{i=1}^{2} p_{i}+P_{c}\right) \\
+\log _{2}\left(1+2 y \sqrt{p_{2} \bar{h}_{2}}-y^{2}\left(p_{1} \bar{h}_{2}+\sigma^{2}\right)\right) \\
\text { s.t. } C_{2}-C_{3}, C_{8}: p_{1} \bar{h}_{1} \geq \sigma^{2} \gamma_{1}^{\min }, \\
C_{9}: p_{2} \bar{h}_{2} \geq p_{1} \bar{h}_{2} \gamma_{2}^{\text {min }}+\sigma^{2} \gamma_{2}^{\min },
\end{gathered}
$$

where $y$ is a non-negative auxiliary variable. $\bar{h}_{1}=h_{1}+\bar{h} \bar{g}_{1}$, $\bar{h}_{2}=h_{2}+\bar{h} \bar{g}_{2}$ and $\bar{h}=\min \left(1, \frac{h_{1}-h_{2}}{\bar{g}_{2}-\bar{g}_{1}}\right)$. For the fixed $p_{i}$, the optimal solution to $y$ is

$$
y^{*}=\frac{\sqrt{p_{2} \bar{h}_{2}}}{p_{1} \bar{h}_{2}+\sigma^{2}} .
$$

which is equivalent to

$$
y^{*}=\left\{\begin{array}{l}
\frac{\sqrt{p_{2}\left(h_{2}+g g_{2}\right)}}{p_{1}\left(h_{2}+g g_{2}\right)+\sigma^{2}}, \rho=1 \\
\frac{\sqrt{p_{2}\left(g_{2} h_{1}-g_{1} h_{2}\right) /\left(g_{2}-g_{1}\right)}}{p_{1}\left(g_{2} h_{1}-g_{1} h_{2}\right) /\left(g_{2}-g_{1}\right)+\sigma^{2}}, \rho=\frac{h_{1}-h_{2}}{g\left(g_{2}-g_{1}\right)}
\end{array}\right.
$$

For the fixed $y, \mathbf{P 5}$ is a convex optimization problem. which can be effectively solved by the CVX tool. However, to further analyze the system performance, we solve it by using Lagrange dual approach to get the closed-form solutions. The Lagrangian function of $\mathbf{P 5}$ can be written by

$$
\begin{aligned}
& L\left(p_{1}, p_{2}, y, \alpha, \beta, \lambda\right)=\log _{2}\left(1+\frac{p_{1} \bar{h}_{1}}{\sigma^{2}}\right)-\eta\left(\sum_{i=1}^{2} p_{i}+P_{c}\right) \\
& +\log _{2}\left(1+2 y \sqrt{p_{2} \bar{h}_{2}}-y^{2}\left(p_{1} \bar{h}_{2}+\sigma^{2}\right)\right)+\alpha\left(P-\sum_{i=1}^{2} p_{i}\right) \\
& +\beta\left(p_{1} \bar{h}_{1}-\sigma^{2} \gamma_{1}^{\text {min }}\right)+\lambda\left(p_{2} \bar{h}_{2}-p_{1} \bar{h}_{2} \gamma_{2}^{\min }-\sigma^{2} \gamma_{2}^{\text {min }}\right)
\end{aligned}
$$

where $\alpha, \beta$ and $\lambda$ are the non-negative Lagrange multipliers. Define the dual objective $D(\alpha, \beta, \lambda)$ as the solution to the following:

$$
D(\alpha, \beta, \lambda)=\max _{p_{1}, p_{2}, y} L\left(p_{1}, p_{2}, y, \alpha, \beta, \lambda\right) .
$$

The dual optimization problem is:

$$
\min _{\alpha, \beta, \lambda} D(\alpha, \beta, \lambda) \text {, s.t. } \alpha \geq 0, \beta \geq 0, \lambda \geq 0 .
$$

According to Karush-Kuhn-Tucker (KKT) conditions [16], the optimal power $p_{1}$ and $p_{2}$ can be calculated by $\partial L / \partial p_{1}=0$ and $\partial L / \partial p_{2}=0$, respectively, i.e.,

$$
\begin{gathered}
p_{1}^{*}=\left[\frac{A_{4}+\sqrt{A_{4}^{2}+4 \bar{h}_{1} \bar{h}_{2} y^{2}\left(A_{1} \sigma^{2}-A_{3} / A_{2}\right)}}{2 \bar{h}_{1} \bar{h}_{2} y^{2}}\right]^{+}, \\
p_{2}^{*}=\left[\frac{B_{2}^{2}+4 B_{3}-B_{2} \sqrt{B_{2}^{2}+8 B_{3}}}{8 B_{1}^{2} \bar{h}_{2}}\right]^{+},
\end{gathered}
$$

where $[x]^{+}=\max (0, x), A_{1}=1+2 y \sqrt{p_{2} h_{2}}-y^{2} \sigma^{2}, A_{2}=$ $\eta+\alpha+\lambda \bar{h}_{2} \gamma_{2}^{\min }-\beta, A_{3}=\bar{h}_{1} A_{1}-y^{2} \bar{h}_{2} \sigma^{2}, A_{4}=\frac{2 \bar{h}_{1} \bar{h}_{2} y^{2}}{A_{2}}+$ 
$y^{2} \bar{h}_{2} \sigma^{2}-\bar{h}_{1} A_{1}, B_{1}=\eta+\alpha-\lambda \bar{h}_{2}, B_{2}=B_{1}\left(1-y^{2}\left(p_{1} \bar{h}_{2}+\right.\right.$ $\left.\left.\sigma^{2}\right)\right)$, and $B_{3}=A_{1} y \bar{h}_{2}$.

Remark 2: For the fixed channel parameters, if $p_{1}$ increases, $B_{2}$ decreases and $p_{2}$ increases based on (20). The reason is that the larger $p_{1}$ means user 2 suffers from severe interference from user 1 , and the transmit power of user 2 has to be increased to overcome the effect of such interference for guaranteeing the minimum QoS requirement of user 2 from $C_{7}$.

Based on the subgradient methods, the Lagrange multipliers can be updated by

$$
\begin{gathered}
\alpha^{t+1}=\left[\alpha^{t}-d_{1}(t) \times\left(P-\sum_{i=1}^{2} p_{i}\right)\right]^{+} \\
\beta^{t+1}=\left[\beta^{t}-d_{2}(t) \times\left(p_{1} \bar{h}_{1}-\sigma^{2} \gamma_{1}^{\min }\right)\right]^{+} \\
\lambda^{t+1}=\left[\lambda^{+}-d_{3}(t) \times\left(p_{2} \bar{h}_{2}-p_{1} \bar{h}_{2} \gamma_{2}^{\min }-\sigma^{2} \gamma_{2}^{\min }\right)\right]^{+}
\end{gathered}
$$

where $t$ denotes the iteration number. $d_{1}(t), d_{2}(t)$ and $d_{3}(t)$ are the positive step sizes at the $t$-th iteration. The algorithm can be guaranteed to converge to the optimal values when the steps are chosen to be sufficiently small [17].

\section{Iterative RA Algorithm Design}

To obtain the optimal power allocation for NOMA users and the optimal RC for the BD user, we propose an iterative algorithm (e.g., Algorithm 1). As shown in Algorithm 1, under the error tolerance $\varepsilon$ and the maximum iteration number $T_{\max }$, we solve $\mathbf{P 5}$ under the fixed $\eta$ and $y$ during each iteration and obtain the optimal solutions by the Lagrange dual approach. The optimal solutions are obtained when $\sum_{i=1}^{2} \log _{2}\left(1+\gamma_{i \rightarrow i}\right)-\eta\left(\sum_{i=1}^{2} p_{i}+P_{c}\right) \leq \varepsilon$ or $t=T_{\max }$ is reached.

\section{Simulation Results}

In this section, we provide numerical results to illustrate the performance of the proposed EE-based BackCom scheme with NOMA users. The simulation settings are as following: the channel gain is the same as [18], $\varepsilon=10^{-6}, \sigma^{2}=-100$ $\mathrm{dBm}, \gamma_{1}^{\text {min }}=\gamma_{2}^{\text {min }}=2 \mathrm{~dB}, P_{c}=1 \mathrm{~mW}$ [19]. The unit bandwidth is considered for simplicity. For performance comparisons, we consider the pure NOMA scheme (e.g., $g=0$, without BackCom) and the pure backscatter scheme with the orthogonal multiple access (OMA) technique.

Fig. 2 shows the total EE of NOMA users versus the channel gain from the $\mathrm{BD}$ to user 2. From the figure, the total $\mathrm{EE}$ of users increases with the increasing channel gain from the $\mathrm{BD}$ to user 2, i.e., $g_{2}^{u}$, when $g_{2}^{u}<g_{1}^{u}$. The RC is $\rho=1$, which does not affect the performance. However, the total EE of users increases when the channel condition between the BD and user 2 is improved. When $g_{2}^{u}>g_{1}^{u}$, the total EE of users is the same for the same $g_{1}^{u}$ because the effect of direct channel gain $g$ is canceled by the expression $\rho * g^{d}$. Moreover, the total EE of users under $g_{1}^{u}=0.45$ is larger than that under $g_{1}^{u}=0.3$ subject to the same channel gain from the BS to the BD.

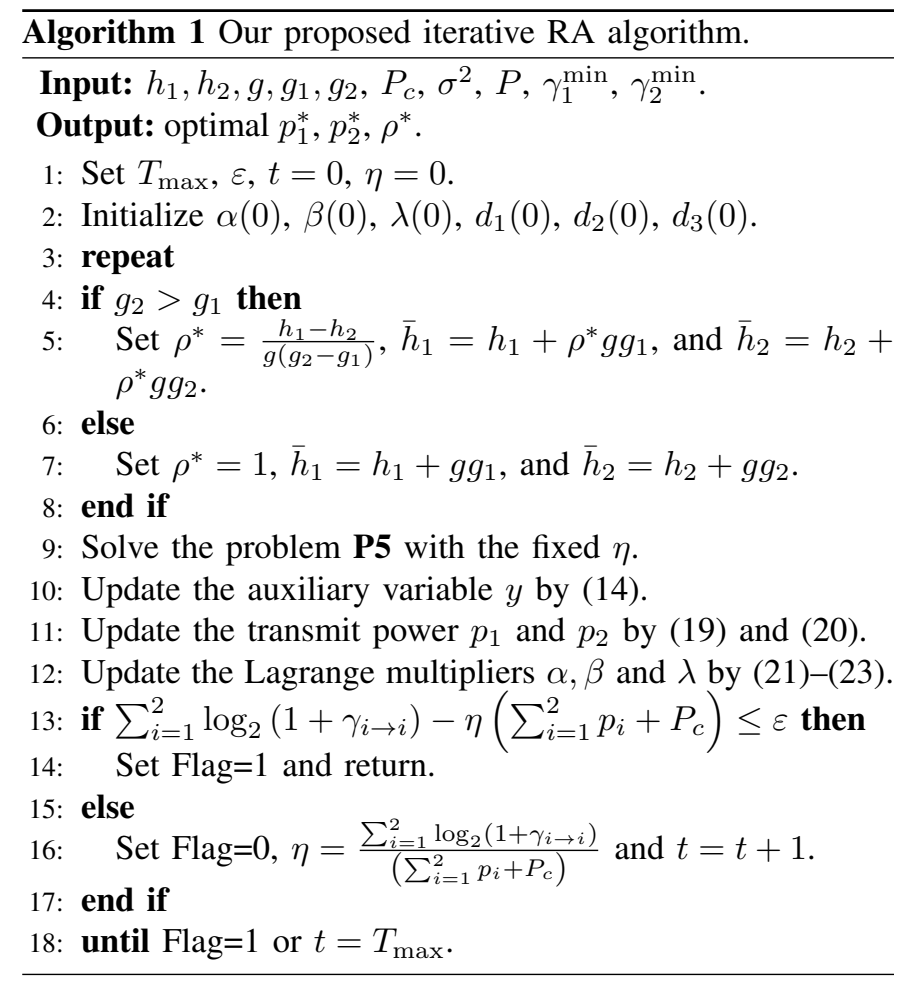

Fig. 3 presents the relationship between the total EE achieved by NOMA users versus the channel gain between BS and user 1 under the fixed $P=1 \mathrm{~W}$. From the figure, the total EE of users increases with the increasing $h_{1}$. Since larger $h_{1}$ refers to higher $\mathrm{RC}$ to improve the backscatter data rate. Furthermore, the proposed algorithm performs the best in terms of EE achieved by considering NOMA and BackCom simultaneously. The OMA scheme is the worst one. Because the NOMA scheme can reduce the co-channel interference by the SIC at the receiver, the EE becomes larger than that of the OMA scheme. Furthermore, the proposed scheme not only considers the NOMA transmission but also the backscatter communication for the high EE.

Fig. 4 shows the total EE of NOMA users versus different maximal transmit power budget at the BS with the fixed $h_{1}=0.5$. From the figure, the proposed scheme achieves the highest EE compared to the pure NOMA scheme and the OMA scheme. Moreover, the total EE degrades with the increasing transmit power budget at the BS. With increasing transmit power budget $P$, the feasible region is enlarged for improving data rates of users. Moreover, the EE of each user decreases with the transmit power budget at the BS.

\section{CONCLUSIONS}

In this paper, we investigated the downlink transmissions of NOMA-based BackCom systems. In order to maximize the total EE of NOMA users, we have formulated it as an optimization problem. By proposing a new iterative algorith$\mathrm{m}$, we have obtained the optimal RC of backscatter device and power allocation for NOMA users. It has shown that the NOMA-based BackCom scheme outperforms the NOMA scheme without backscatter way, and the OMA scheme in 


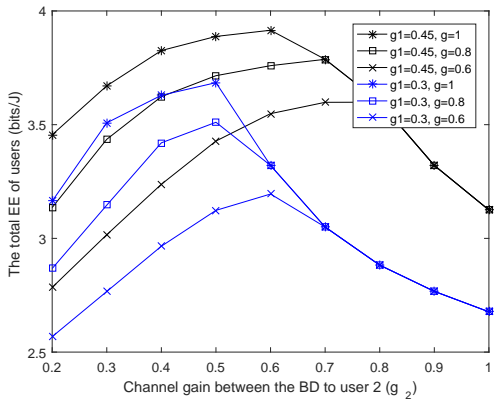

Fig. 2. Total EE versus channel gain $g_{2}$.

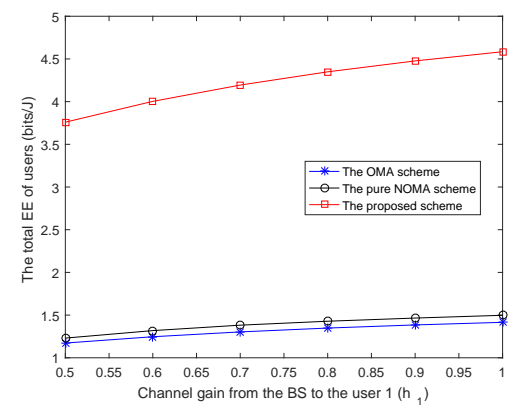

Fig. 3. Total EE versus channel gain $h_{1}$.

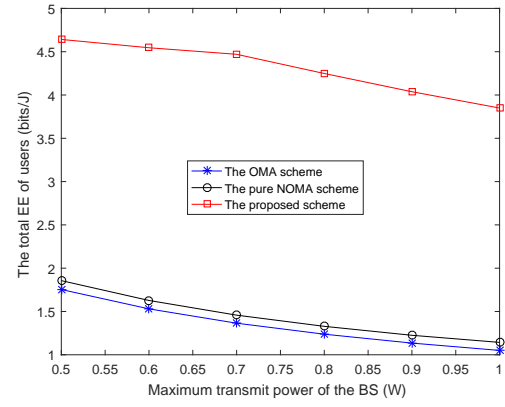

Fig. 4. Total EE versus transmit power $P$. terms of EE, which could be used to extend the network lifetime.

\section{APPENDIX A}

The PRoof of Proposition 1

Define $F=F_{1}+F_{2}, F_{1}(\rho)=\log _{2}\left(1+\frac{p_{1}\left(h_{1}+\rho \bar{g}_{1}\right)}{\sigma^{2}}\right), A=$ $p_{1}\left(h_{2}+\rho \bar{g}_{2}\right)+\sigma^{2}$ and $F_{2}(\rho)=\log _{2}\left(1+\frac{p_{2}\left(h_{2}+\rho \bar{g}_{2}\right)}{p_{1}\left(h_{2}+\rho \bar{g}_{2}\right)+\sigma^{2}}\right)$, thus we have the first-order derivations, i.e.,

$$
\begin{array}{r}
\frac{\partial F_{1}}{\partial \rho}=\frac{p_{1} \bar{g}_{1}}{\ln 2\left(p_{1} h_{1}+p_{1} \rho \bar{g}_{1}+\sigma^{2}\right)}>0, \\
\frac{\partial F_{2}}{\partial \rho}=\frac{p_{2} \bar{g}_{2} \sigma^{2}}{\ln 2\left(A^{2}+A\left(p_{2} h_{2}+p_{2} \rho \bar{g}_{2}\right)\right)}>0 .
\end{array}
$$

Thus, we have the second-order derivations, i.e.,

$$
\begin{gathered}
\frac{\partial^{2} F_{1}}{\partial \rho^{2}}=-\frac{\left(p_{1} \bar{g}_{1}\right)^{2}}{\ln 2\left(p_{1} h_{1}+p_{1} \rho \bar{g}_{1}+\sigma^{2}\right)^{2}}<0, \\
\frac{\partial^{2} F_{2}}{\partial \rho^{2}}=-\frac{p_{2} \sigma^{2} \bar{g}_{2}^{2}\left(A\left(2 p_{1}+p_{2}\right)+p_{1} p_{2}\left(h_{2}+\rho \bar{g}_{2}\right)\right)}{\ln 2\left(A^{2}+A\left(p_{2} h_{2}+p_{2} \rho \bar{g}_{2}\right)\right)^{2}}<0 .
\end{gathered}
$$

Therefore, $F$ is a concave function with respective to $\rho$ and an increasing function with the variable $\rho \in[0,1]$. As a result, the maximum value of the objective function in $\mathbf{P 2}$ can be determined by the upper bound of the RC. According to $C_{5^{-}}$. $C_{7}$, we have $\rho \leq \frac{h_{1}-h_{2}}{g^{d}\left(g_{2}^{u}-g_{1} u\right)}$ if $g_{2}^{u}>g_{1}^{u}$. Accordingly, the optimal RC is $\rho^{*}=\frac{h_{1}-h_{2}}{g^{d}\left(g_{2}^{u}-g_{1}^{u}\right)}$ when $h_{1}-h_{2}<g^{d}\left(g_{2}^{u}-g_{1}^{u}\right)$. When $h_{1}-h_{2}>g^{d}\left(g_{2}^{u}-g_{1}^{u}\right)$, the optimal value is $\rho^{*}=1$. When $g_{2}^{u}<g_{1}^{u}$, the constraint $C_{5}$ is always established since $\rho \geq 0$. Under this case, the optimal RC is $\rho^{*}=1$.

\section{APPENDIX B}

\section{The PRoOF OF PROPOSITION 2}

According to the first-order derivation, we have $\frac{\partial G}{\partial p_{2}}=$ $\frac{\bar{h}_{2}}{\left(p_{1}+p_{2}\right) h_{2}+\sigma^{2}}$ and $\frac{\partial G}{\partial p_{1}}=\frac{\bar{h}_{2}}{\left(p_{1}+p_{2}\right) h_{2}+\sigma^{2}}-\frac{\bar{h}_{2}}{p_{1} h_{2}+\sigma^{2}}$. Thus, the second-order derivations are

$\frac{\partial^{2} G}{\partial p_{2}^{2}}=\frac{\partial^{2} G}{\partial p_{2} \partial p_{1}}=\frac{\partial^{2} G}{\partial p_{1} \partial p_{2}}=-\frac{\bar{h}_{2}^{2}}{\ln 2\left(\left(p_{1}+p_{2}\right) \bar{h}_{2}+\sigma^{2}\right)^{2}}<0$,

$$
\frac{\partial^{2} G}{\partial p_{1}^{2}}=\frac{p_{2} \bar{h}_{2}^{3}\left(p_{2} \bar{h}_{2}+2\left(p_{1} \bar{h}_{2}+\sigma^{2}\right)\right)}{\ln 2\left(\left(p_{1} \bar{h}_{2}+\sigma^{2}\right)\left(p_{1} \bar{h}_{2}+\sigma^{2}+p_{2} \bar{h}_{2}\right)\right)^{2}}>0 .
$$

Therefore, $G(\cdot)$ is a non-convex or non-concave function, which is the difference of two concave function (i.e., D.C. function), e.g., $G=\log _{2}\left(1+\left(p_{1}+p_{2}\right) \bar{h}_{2}+\sigma^{2}\right)-\log _{2}(1+$ $\left.p_{1} \bar{h}_{2}+\sigma^{2}\right)$.

\section{REFERENCES}

[1] Y. Kawamoto, et al., "Efficient resource allocation utilizing Q-learning in multiple UA communications," IEEE Trans. Netw. Sci. Eng., vol. 6, no. 3, pp. 293-302, Mar. 2019

[2] N. Van Huynh, et al., "Ambient backscatter communications: a contemporary survey," IEEE Commun. Surveys \& Tuts., vol. 20, no. 4, pp. 2889-2922, Oct. 2018.

[3] B. Lyu, et al., "Relay cooperation enhanced backscatter communication for internet-of-things," IEEE Internet Things J., vol. 6, no. 2, pp. 28602871, Apr. 2019.

[4] Y. Liu, et al., "Non-orthogonal multiple access for 5G and beyond," Proc. IEEE, vol. 105, no. 12, pp. 2347-2381, Dec. 2017.

[5] G. Gui, H. Sari, and E. Biglieri, "A new definition of fairness for nonorthogonal multiple access," IEEE Commun. Lett., vol. 23, no. 7, pp. 1267-1271, May 2019.

[6] J. Guo, X. Zhou, S. Durrani and H. Yanikomeroglu, "Backscatter communications with NOMA," Proc. ISWCS, Lisbon, 2018, pp. 1-5.

[7] Q. Zhang, L. Zhang, Y. Liang and P. Kam, "Backscatter-NOMA: a symbiotic system of cellular and internet-of-things networks," IEEE Access, vol. 7, pp. 20000-20013, Feb. 2019.

[8] C. Le and D. Do, "Outage performance of backscatter NOMA relaying systems equipping with multiple antennas," Electronics Lett., vol. 55, no. 19, pp. 1066-1067, 2019.

[9] J. Guo, et al., "Design of non-orthogonal multiple access enhanced backscatter communication," IEEE Trans. Wireless Commun., vol. 17, no. 10, pp. 6837-6852, Oct. 2018.

[10] G. Yang, X. Xu and Y. Liang, "Resource allocation in NOMA-enhanced backscatter communication networks for wireless powered IoT," IEEE Wireless Commun. Lett., doi: 10.1109/LWC.2019.2944369.

[11] H. Zhang, et al., "Energy efficient resource management in SWIPT enabled heterogeneous networks with NOMA," IEEE Trans. Wireless Commun., doi: 10.1109/TWC.2019.2948874.

[12] M. Liu, G. Gui, N. Zhao, J. Sun, G. Haris, H. Sari, "UAV-aided airto-ground cooperative non-orthogonal multiple access," IEEE Internet Things J., doi: 10.1109/JIOT.2019.2957225

[13] Z. Ding, et al., "A survey on non-orthogonal multiple access for 5G networks: research challenges and future trends," IEEE J. Sel. Areas Commun., vol. 35, no. 10, pp. 2181-2195, Oct. 2017.

[14] Y. Liu, et al., "Multiple-antenna-assisted non-orthogonal multiple access," IEEE Wireless Commun., vol. 25, no. 2, pp. 17-23, Apr. 2018.

[15] K. Shen and W. Yu, "Fractional programming for communication systems-part I: power control and beamforming," IEEE Trans. Signal Process., vol. 66, no. 10, pp. 2616-2630, May 2018.

[16] S. Boyd and L. Vandenberghe, Convex Optimization. Cambridge Univ., 2004.

[17] D. Bertsekas, Nonlinear Programming. Belmont, MA: Athena Scientific, 1999.

[18] P. Setoodeh and S. Haykin, "Robust transmit power control for cognitive radio," Proc. IEEE, vol. 97, no. 5, pp. 915-939, May 2009.

[19] S. Yang, et al., "Energy efficiency optimization for UAV-assisted backscatter communications," IEEE Commun. Lett., vol. 23, no. 11, pp. 2041-2045, Nov. 2019. 